\title{
Follow-up observations of X-ray emitting hot subdwarf stars: the He-rich sdO BD $+37^{\circ} 1977$
}

\author{
N. La Palombara ${ }^{1}$, P. Esposito ${ }^{1,2}$, S. Mereghetti ${ }^{1}$, G. Novara ${ }^{1,3}$, and A. Tiengo ${ }^{1,3,4}$ \\ ${ }^{1}$ INAF, Istituto di Astrofisica Spaziale e Fisica Cosmica - Milano, via Bassini 15, 20133 Milano, Italy \\ e-mail: nicola@iasf-milano.inaf.it \\ 2 Harvard-Smithsonian Center for Astrophysics, 60 Garden Street, Cambridge, MA 02138, USA \\ 3 IUSS-Istituto Universitario di Studi Superiori, piazza della Vittoria 15, 27100 Pavia, Italy \\ ${ }^{4}$ Istituto Nazionale di Fisica Nucleare, Sezione di Pavia, via A. Bassi 6, 27100 Pavia, Italy
}

Received 20 March 2015 / Accepted 19 June 2015

\begin{abstract}
We report on the results of the first XMM-Newton satellite observation of the luminous and helium-rich O-type subdwarf $\mathrm{BD}+37^{\circ} 1977$ carried out in April 2014. X-ray emission is detected with a flux of about $4 \times 10^{-14} \mathrm{erg} \mathrm{cm}^{-2} \mathrm{~s}^{-1}(0.2-1.5 \mathrm{keV})$, corresponding to a $f_{\mathrm{X}} / f_{\text {bol }}$ ratio $\sim 10^{-7}$; the source spectrum is very soft, and is well fit by the sum of two plasma components at different temperatures. Both characteristics are in agreement with what is observed in the main-sequence early-type stars, where the observed X-ray emission is due to turbulence and shocks in the stellar wind. A smaller but still significant stellar wind has also been observed in $\mathrm{BD}+37^{\circ} 1977$; therefore, we suggest that in this case the detected X-ray flux has the same origin.
\end{abstract}

Key words. stars: early-type - stars: individual: BD $+37^{\circ} 1977$ - subdwarfs - X-rays: stars

\section{Introduction}

Among the hot subdwarf (sd) stars, which are evolved He-core burning low-mass stars (Heber 2009), the sdO stars are those that show the highest temperatures $\left(T_{\text {eff }}>40 \mathrm{kK}\right)$. Apart from this characteristic, sdO stars (sdOs) are characterized by a wide range of values for the surface gravity $(\log (g)=4-6.5)$ and helium abundance $\left(-3.5 \lesssim \log \left(n_{\mathrm{He}} n_{\mathrm{H}}^{-1}\right) \lesssim 3\right)$. They form a rather heterogeneous class of stars, which includes both He-poor and He-rich stars (Heber \& Jeffery 1992; Heber et al. 2006; Hirsch et al. 2008), and luminous and compact stars, according to their low or high surface gravity, respectively (Napiwotzki 2008). This variety of properties is probably the consequence of different evolutionary histories (Heber 2009; Geier 2015): in the case of the compact stars, the He-poor ones are post-EHB stars, while the origin of the He-rich ones might be either the merging of two He-core or C/O-core white dwarfs (Iben 1990; Saio \& Jeffery 2000, 2002) or the so-called late hot-flasher scenario (Brown et al. 2001); instead, the luminous sdO stars are postAGB stars. Evolutionary models suggest that most sdO stars are the outcome of the evolution of single stars, but some of them could descend from binary systems that underwent a commonenvelope phase; in this case it is possible that the sdO stars have a compact companion, typically a white dwarf (WD).

Up to now, sdO stars have been deeply investigated in the optical/UV domain, where several of them are rather bright; on the other hand, only few of them are known as X-ray sources. In the case of the binary HD 49798, the detection of pulsed $(P=13.18 \mathrm{~s})$ soft $\mathrm{X}$-rays (Israel et al. 1997) indicates that this emission originates from accretion onto a compact object, most likely a massive WD (Mereghetti et al. 2009). For this binary we also detected an evident X-ray emission when the compact companion is eclipsed by the sdO star, suggesting the possibility of intrinsic X-ray emission of the sdO star (Mereghetti et al. 2013).
Another sdO star recently detected at X-rays is $\mathrm{BD}+37^{\circ} 442$ : the XMM-Newton observation of this He-rich star revealed soft X-ray emission, with a spectrum similar to that of HD 49798, and a possible periodicity of $19.16 \mathrm{~s}$ (at $3 \sigma$ confidence level), which suggests that $\mathrm{BD}+37^{\circ} 442$ also has a compact companion (La Palombara et al. 2012). In order to enlarge the sample of sdO stars observed at X-rays, we performed with Chandra HRC-I a survey of a complete flux-limited sample of sdO stars and discovered three additional X-ray emitting stars (La Palombara et al. 2014): the luminous and He-rich sdO star BD $+37^{\circ} 1977$ (Jeffery \& Hamann 2010), and the compact $(\log (g)>6)$ and He-poor stars Feige 34 and BD+28 4211 (Thejll et al. 1991; Zanin \& Weinberger 1997).

In this paper we report on the results of a follow-up observation of $\mathrm{BD}+37^{\circ} 1977$, performed with XMM-Newton, which allowed us to investigate in detail the spectral and timing properties of the X-ray emission discovered with Chandra. This star was identified as an sdO star by Wolff et al. (1974), who detected several emission $\mathrm{He}$ lines but no $\mathrm{H}$ lines in its blue spectrum. Their spectroscopic analysis gave a surface gravity $\log g \lesssim 4.5$ and a temperature $T \lesssim 50 \mathrm{kK}$; comparable values ( $T \simeq 55 \mathrm{kK}$ and $\log g \simeq 4.0$ ) were estimated from the low resolution IUE spectrum (Darius et al. 1979), which also gave an estimate of the star luminosity $\left(\log \left(L_{\mathrm{bol}} / L_{\odot}=4.4\right)\right)$. There is no evidence for a compact companion for $\mathrm{BD}+37^{\circ} 1977$. The possible detection of an infrared excess at $2 \sigma$ confidence level (Ulla \& Thejll 1998), if confirmed, could imply a companion star of spectral type earlier than G4. Based on high-resolution ultraviolet and optical spectra and on UV-optical-IR photometry, and using the latest generation of models for spherically expanding stellar atmospheres, Jeffery \& Hamann (2010) found that $\mathrm{BD}+37^{\circ} 1977$ is characterized by a significant mass-loss rate $\left(\dot{M}=10^{-8.2} M_{\odot} \mathrm{y}^{-1}\right)$. Their analysis yielded revised and better constrained spectral parameters, very similar to those of 
A\&A 580, A56 (2015)

Table 1. Main parameters of the sdO stars BD $+37^{\circ} 1977, \mathrm{BD}+37^{\circ} 442$, and $\mathrm{HD} 49798$.

\begin{tabular}{|c|c|c|c|c|c|c|c|}
\hline \multirow[t]{2}{*}{ Parameter } & \multirow[t]{2}{*}{ Symbol } & \multicolumn{2}{|c|}{$\mathrm{BD}+37^{\circ} 1977$} & \multicolumn{2}{|c|}{$\mathrm{BD}+37^{\circ} 442$} & \multicolumn{2}{|c|}{ HD 49798} \\
\hline & & Value & Reference & Value & Reference & Value & Reference \\
\hline Surface gravity & $\log g$ & $\simeq 4.0$ & 1 & $4.00 \pm 0.25$ & 4 & 4.35 & 6 \\
\hline Luminosity $\left(L_{\odot}\right)$ & $L$ & 25000 & 1 & 25000 & 1 & 14000 & 6 \\
\hline Effective temperature $(\mathrm{K})$ & $T_{\text {eff }}$ & 48000 & 2 & 48000 & 2 & 46500 & 6 \\
\hline \multirow[t]{3}{*}{ Magnitudes } & $U$ & 8.67 & 3 & 8.57 & 5 & 6.76 & 7 \\
\hline & $B$ & 9.93 & 3 & 9.73 & 5 & 8.02 & 7 \\
\hline & $V$ & 10.17 & 3 & 10.01 & 5 & 8.29 & 7 \\
\hline Distance $(\mathrm{kpc})$ & $d$ & $\simeq 2.7$ & 2 & $2.0_{-0.6}^{+0.9}$ & 4 & $0.65 \pm 0.10$ & 8 \\
\hline Terminal wind velocity $\left(\mathrm{km} \mathrm{s}^{-1}\right)$ & $v_{\infty}$ & 2000 & 2 & 2000 & 2 & 1350 & 9 \\
\hline Mass-loss rate $\left(M_{\odot} \mathrm{yr}^{-1}\right)$ & $\dot{M}$ & $10^{-8.2}$ & 2 & $10^{-8.5}$ & 2 & $10^{-8.5}$ & 6 \\
\hline
\end{tabular}

References. 1) Darius et al. (1979); 2) Jeffery \& Hamann (2010); 3) Jordi et al. (1991); 4) Bauer \& Husfeld (1995); 5) Landolt (1973); 6) Hamann (2010); 7) Landolt \& Uomoto (2007); 8) Kudritzki \& Simon (1978); 9) Hamann et al. (1981).

Table 2. Observations of BD $+37^{\circ} 1977$ performed by XMM-Newton.

\begin{tabular}{|c|c|c|c|c|}
\hline \multirow[t]{2}{*}{ Revolution } & \multirow[t]{2}{*}{ Observation ID } & \multirow{2}{*}{$\begin{array}{l}\text { Start time } \\
\text { (YYYY-MM-DD hh:mm:ss) }\end{array}$} & \multicolumn{2}{|c|}{ Effective exposure } \\
\hline & & & $p n(\mathrm{ks})$ & $\operatorname{MOS}(\mathrm{ks})$ \\
\hline 2627 & 0740140301 & 2014-04-14@15:00:25 & 4.2 & 8.5 \\
\hline 2629 & 0740140501 & 2014-04-18@14:43:21 & 7.0 & 11.3 \\
\hline 2630 & 0740140401 & 2014-04-20@15:45:37 & 7.9 & 10.0 \\
\hline 2631 & 0740140601 & 2014-04-22@14:36:30 & 9.9 & 14.4 \\
\hline 2632 & 0740140701 & 2014-04-24@14:06:44 & 5.5 & 6.8 \\
\hline
\end{tabular}

$\mathrm{BD}+37^{\circ} 442$, the other X-ray detected He-rich sdO star. The best-fit distance modulus obtained from this analysis is $D M=$ 12.2 , corresponding to a distance of $\simeq 2.7 \mathrm{kpc}$. Our observation of $\mathrm{BD}+37^{\circ} 1977$ with Chandra HRC-I provided a detection with a count rate $\mathrm{CR}=3.6_{-0.9}^{+1.1} \mathrm{cts} \mathrm{s}^{-1}$, which - assuming a spectrum similar to that of $\mathrm{HD} 49798$ and $\mathrm{BD}+37^{\circ} 442$ - implies an X-ray luminosity $L_{X} \sim 10^{31} \mathrm{erg} \mathrm{s}^{-1}$. For comparison, in Table 1 we list the main parameters of the three luminous sdO stars detected in $\mathrm{X}$-rays.

\section{Observations and data analysis}

BD $+37^{\circ} 1977$ was observed with XMM-Newton in April 2014. At that time the source was visible only for the first $\sim 20 \mathrm{ks}$ of each XMM-Newton orbit: therefore, five different observations were performed between April 14 and April 24 (see Table 2). The three EPIC cameras, i.e. one pn (Strüder et al. 2001) and two MOS (Turner et al. 2001), were always operated in full frame mode, with time resolution of $73 \mathrm{~ms}$ for the $p n$ and $2.6 \mathrm{~s}$ for the two MOS cameras; taking into account all the observations, the total effective exposure time was, respectively, of $\simeq 34.5 \mathrm{ks}$ and $\simeq 50 \mathrm{ks}$. For all cameras the medium thickness filter was used.

We used version 13.5 of the XMM-Newton Science Analysis System (SAS) to process the event files. For the data analysis we selected only the events with pattern in the range 0-4 (i.e. mono- and bi-pixel events) for the $p n$ camera and 0-12 (i.e. from 1 to 4 pixel events) for the two MOS. For each camera, we merged the data of the five observations and accumulated the images in various energy ranges. We found that $\mathrm{BD}+37^{\circ} 1977$ is significantly detected at the coordinates $\mathrm{RA}=9^{\mathrm{h}} 24^{\mathrm{m}} 26.4^{\mathrm{s}}$, Dec $=+36^{\circ} 42^{\prime} 52.8^{\prime \prime}$, which differ by $0.7^{\prime \prime}$ from the position of $\mathrm{BD}+37^{\circ} 1977$. This difference is consistent with the $\sim 2^{\prime \prime} \mathrm{rms}$ astrometric accuracy of XMM-Newton ${ }^{1}$. In each of the five observations a point source is clearly detected below $0.5 \mathrm{keV}$, while considering the five merged observations the source is detected up to $\sim 1.5 \mathrm{keV}$ (Fig. 1). This implies that the source spectrum

\footnotetext{
1 http://xmm2.esac.esa.int/docs/documents/

CAL-TN-Q018.ps.gz
}

is very soft. All the observations were partly affected by high instrumental background. However, since the spectrum of the instrumental background is rather hard, the background contamination has a limited impact on the source spectral analysis; therefore, we considered the whole data set without rejecting the time intervals with the highest particle background. The source net count rate in the $0.15-1.5 \mathrm{keV}$ range is $(1.7 \pm 0.2) \times 10^{-2} \mathrm{cts} \mathrm{s}^{-1}$ and $(2.5 \pm 0.3) \times 10^{-3} \mathrm{cts} \mathrm{s}^{-1}$ for the $p n$ and both of the two MOS, respectively.

For the timing and spectral analysis, we used the data of the whole observation and the three EPIC cameras; we extracted the source events from a circular region with radius $15^{\prime \prime}$ centred at the source position, while the corresponding background events were accumulated on circular areas free of sources and radii of $30^{\prime \prime}$ and $120^{\prime \prime}$ for the $p n$ and the two MOS cameras, respectively. We converted the arrival times to the solar system barycentre, then we combined the three data sets in a single event list. The background-subtracted light curve of $\mathrm{BD}+37^{\circ} 1977$ does not show any variability on time scales from hundreds of seconds to the observation length. We looked for possible periodicities in the X-ray emission, but we found no evidence of periodic signals; this search was unsuccessful not only for the five individual observations, but also when considering all of them together. In all cases we estimated an upper limit of $\sim 30 \%$ on the pulsed fraction, for a sinusoidal modulation between $1 \mathrm{~s}$ and $5000 \mathrm{~s}$.

For the spectral analysis we considered first the $p n$ data, since the source soft spectrum and the lower sensitivity of the MOS cameras at low energies strongly reduced the count statistics. We verified that the addition of the MOS data gave consistent results. We generated the response matrix and ancillary file using the SAS tasks rmfgen and arfgen. To ensure the applicability of the $\chi^{2}$ statistics, the spectrum was rebinned with a minimum of 30 net counts per bin; then we fitted them using XSPEC (V 12.7.0). We only used the energy range $0.2-1.5 \mathrm{keV}$ since at higher energies the background dominates and the source flux is negligible. In the following, all the spectral uncertainties and upper limits are given at the $90 \%$ confidence level for one interesting parameter, and we assume a source distance 

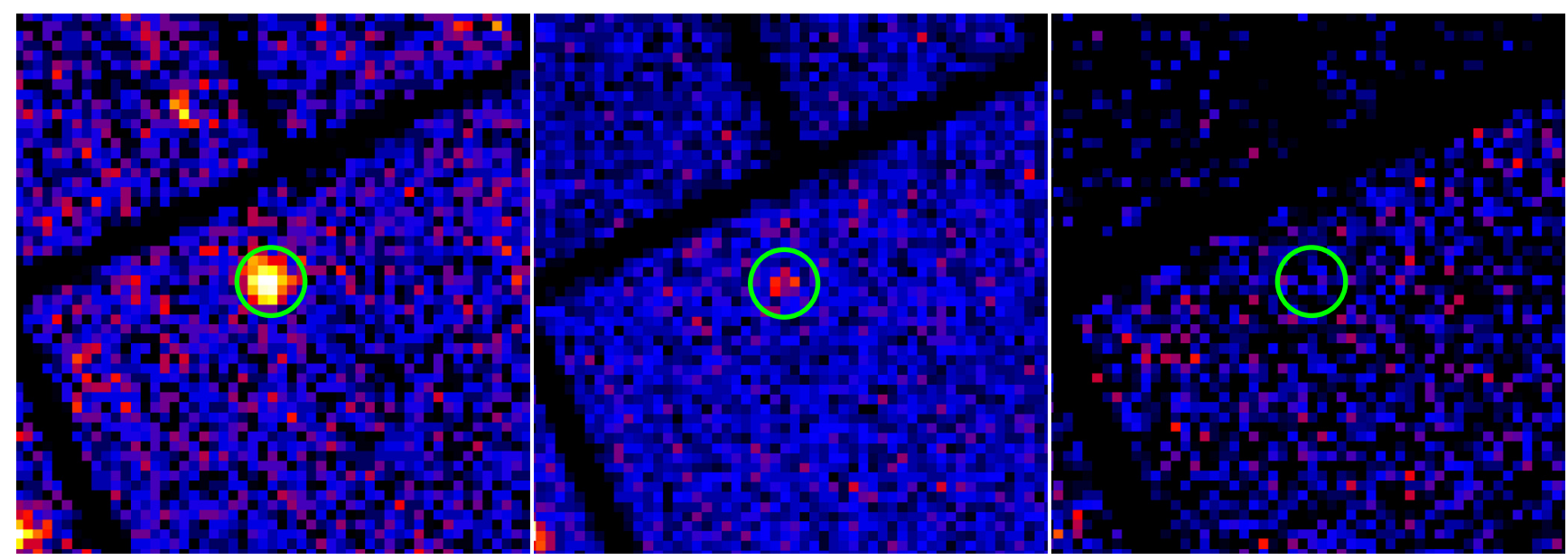

Fig. 1. EPIC $p n$ image of the sky region around $\mathrm{BD}+37^{\circ} 1977$ in the energy ranges $0.15-0.5$ (left), $0.5-1.5$ (centre), and $1.5-10 \mathrm{keV}$ (right). The green circle (15" radius) indicates the source position.

of $2.7 \mathrm{kpc}$ (Jeffery \& Hamann 2010); we adopted the results of Anders \& Grevesse (1989) for the solar abundances of the atomic elements.

The source spectrum is very soft and we tried to describe it with different models (see Table 3). The fit with an absorbed power law (PL) is formally acceptable $\left(\chi_{v}^{2}<2\right)$, but gives a very large and unrealistic photon index $(\Gamma \simeq 5)$, while a fit with a blackbody model is rejected by the data $\left(\chi_{v}^{2}>2\right)$. An absorbed power law plus blackbody gives a good fit, but with unrealistic values (for the power-law photon index) or unconstrained values (for the blackbody normalization) of the model parameters. We note that while this model is physically motivated for $\mathrm{BD}+37^{\circ} 442$, where the observed X-ray flux can be attributed to accretion onto a compact companion, this is not the case for $\mathrm{BD}+37^{\circ} 1977$, for which no evidence of a compact companion has been found. For this reason, we consider in the following the possibility that the $\mathrm{X}$-ray emission detected in $\mathrm{BD}+37^{\circ} 1977$ has the same origin as that observed in the normal, giant, and supergiant early-type $\mathrm{O}$ stars.

For a large sample of this type of stars observed with $X M M-N e w t o n$ the spectrum can be described by the sum of different thermal plasma components (MEKAL in XSPEC), with temperatures between $\simeq 0.1$ and $\simeq 5 \mathrm{keV}$ (Nazé 2009). Therefore, we tried to use the same approach also in the case of BD $+37^{\circ} 1977$. We clearly found that, assuming solar abundances, with this model it was not possible to obtain an acceptable fit, even if we considered the sum of two MEKAL components at different temperatures (Fig. 2 and Table 3). Therefore, we modified the model abundances by taking into account the values of the single chemical elements considered by Jeffery \& Hamann (2010). Since there are no abundance measurements for BD $+37^{\circ} 1977$, they adopted the same overabundance of $\mathrm{He}, \mathrm{C}, \mathrm{N}, \mathrm{Si}$, and $\mathrm{Fe}$ obtained by Bauer \& Husfeld (1995) for BD $+37^{\circ} 442$, which is very similar from the spectroscopic point of view.

Assuming these abundances, a single thermal component provides an acceptable fit $\left(\chi_{v}^{2}<2\right)$ but leaves large residuals at the high energies. Hence, we considered a model composed of the sum of two absorbed components at different temperatures. We checked that the estimated interstellar absorption is negligible and consistent with 0 . Therefore, we fixed it at $N_{\mathrm{H}}=$ $10^{20} \mathrm{~cm}^{-2}$, which is the total absorption value across the Galaxy in the direction of $\mathrm{BD}+37^{\circ} 1977$. In this way we found a good fit $\left(\chi_{v}^{2}=0.71\right.$ for 13 degrees of freedom, Fig. 3) with $k T_{1}=120 \pm$ $30 \mathrm{eV}$ and $k T_{2}=840_{-210}^{+350} \mathrm{eV}$. The total flux in the energy range

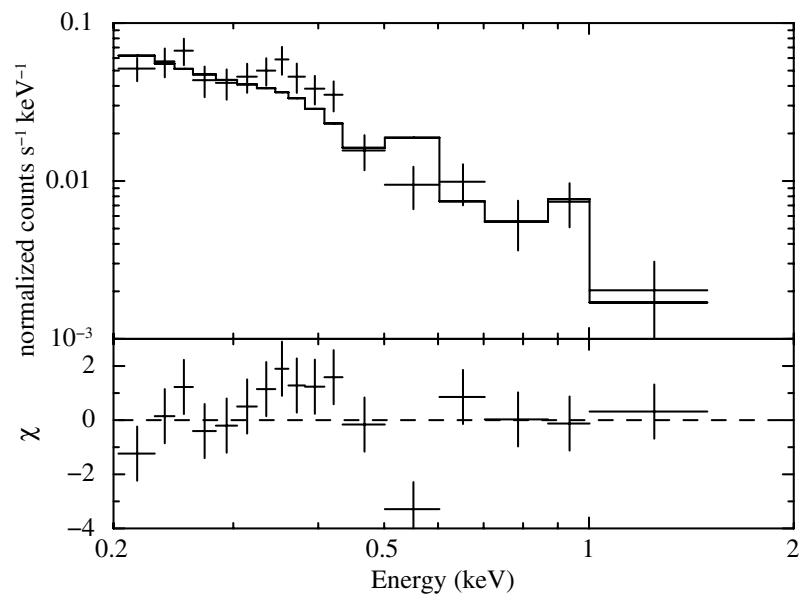

Fig. 2. Top panel: EPIC pn spectrum of $\mathrm{BD}+37^{\circ} 1977$ with the best-fit model composed of the sum of two thermal plasma components, with solar abundances. Bottom panel: residuals (in units of $\sigma$ ) between data and model.

$0.2-1.5 \mathrm{keV}$ is $f_{\mathrm{X}}=\left(4.0_{-0.3}^{+0.2}\right) \times 10^{-14} \mathrm{erg} \mathrm{cm}^{-2} \mathrm{~s}^{-1}$; although $78 \%$ of the flux is due to the low-temperature component, the high-temperature component is significant at $3 \sigma$ confidence level. The measured flux corresponds to a source luminosity $L_{\mathrm{X}}=\left(3.3_{-0.3}^{+0.2}\right) \times 10^{31} \mathrm{erg} \mathrm{s}^{-1}$. The fit leaves some residuals at $\sim 650 \mathrm{eV}$, thus suggesting the presence of the $\mathrm{O}$ VIII emission line (Fig. 3). This could be due to an underestimation of the real oxygen abundance since in our model we fixed it at the solar value. Therefore, we repeated the spectral fit with the same model but leaving the oxygen abundance free to vary. In this way we obtained an improvement of the spectral fit (Fig. 4) and we found that the best-fit abundance value is $310 \pm 250$ times the solar value; although it is not well constrained, it is consistent with the expected oxygen overabundance in BD $+37^{\circ} 1977$.

\section{Discussion}

The XMM-Newton observation of $\mathrm{BD}+37^{\circ} 1977$ enabled us to constrain the flux and spectrum of the X-ray emission recently discovered by Chandra (La Palombara et al. 2014). The measured flux $f_{\mathrm{X}} \simeq 4 \times 10^{-14} \mathrm{erg} \mathrm{cm}^{-2} \mathrm{~s}^{-1}$ confirms the estimate provided by the Chandra detection. It implies a luminosity $L_{\mathrm{X}} \simeq 3.3 \times 10^{31} \mathrm{erg} \mathrm{s}^{-1}$. Since the bolometric luminosity of 
Table 3. Summary of the best-fit parameters of BD $+37^{\circ} 1977$ obtained with different spectral models.

\begin{tabular}{ccc}
\hline \hline Parameter & Unit & Value \\
\hline \multicolumn{4}{c}{ Power law } \\
$N_{\mathrm{H}}$ & $\mathrm{cm}^{-2}$ & $\left(3.5_{-2.7}^{+4.0}\right) \times 10^{20}$ \\
$\Gamma$ & - & $5.0_{-1.1}^{+1.7}$ \\
$\chi_{v}^{2}$ & - & 1.76 \\
Degrees of freedom & - & 14 \\
\hline \multicolumn{4}{c}{ Blackbody } \\
$N_{\mathrm{H}}$ & $\mathrm{cm}^{-2}$ & $\left(0.9_{-0.9}^{+5.8}\right) \times 10^{20}$ \\
$k T$ & $\mathrm{eV}$ & $67_{-22}^{+12}$ \\
$\chi_{v}^{2}$ & - & 2.46 \\
Degrees of freedom & - & 14 \\
\hline \multicolumn{4}{c}{ Power law + Blackbody } \\
$N_{\mathrm{H}}$ & $\mathrm{cm}^{-2}$ & $\left(2.0_{-1.3}^{+2.0}\right) \times 10^{21}$ \\
$\Gamma_{\mathrm{PL}}$ & - & $3.9_{-1.7}^{+2.3}$ \\
$k T_{\mathrm{BB}}$ & $\mathrm{eV}$ & $22_{-12}^{+16}$ \\
$\chi_{v}^{2}$ & - & 0.76 \\
Degrees of freedom & - & 12 \\
\hline \multicolumn{2}{c}{}
\end{tabular}

Mekal + Mekal

(with solar abundances)

\begin{tabular}{ccc}
\multicolumn{4}{c}{ (with solar abundances) } \\
$N_{\mathrm{H}}$ & $\mathrm{cm}^{-2}$ & $(1.8 \pm 1.2) \times 10^{20}$ \\
$k T_{1}$ & $\mathrm{eV}$ & $81_{-0}^{+5}$ \\
$k T_{2}$ & $\mathrm{eV}$ & $800_{-230}^{+330}$ \\
$\chi_{v}^{2}$ & - & 2.15 \\
Degrees of freedom & - & 12 \\
\hline \multicolumn{4}{c}{ Mekal + Mekal } \\
(with abundances from Jeffery \& Hamann 2010) \\
$N_{\mathrm{H}}$ & $\mathrm{cm}^{-2}$ & $1 \times 10^{20}($ fixed) \\
$k T_{1}$ & $\mathrm{eV}$ & $120 \pm 30$ \\
$k T_{2}$ & $\mathrm{eV}$ & $840_{-210}^{+350}$ \\
$\chi_{v}^{2}$ & - & 0.71 \\
Degrees of freedom & - & 13 \\
\hline
\end{tabular}

Mekal + Mekal

(with abundances from Jeffery \& Hamann 2010) (with free Oxygen abundance)

\begin{tabular}{lcc}
$N_{\mathrm{H}}$ & $\mathrm{cm}^{-2}$ & $1 \times 10^{20}$ (fixed) \\
$k T_{1}$ & $\mathrm{eV}$ & $100_{-20}^{+40}$ \\
$k T_{2}$ & $\mathrm{eV}$ & $840_{-250}^{+570}$ \\
Abundance & - & $310 \pm 250$ \\
$\chi_{v}^{2}$ & - & 0.64 \\
s of freedom & - & 12 \\
\hline
\end{tabular}

$\mathrm{BD}+37^{\circ} 1977$ is $L_{\mathrm{bol}} \simeq 25000 L_{\odot}$ (Darius et al. 1979 ; Jeffery \& Hamann 2010), the corresponding ratio is $L_{\mathrm{X}} / L_{\mathrm{bol}} \sim 10^{-6.5}$. This value is consistent with the canonical relation $L_{\mathrm{X}} \sim 10^{-7} \times L_{\mathrm{bol}}$ obtained for the normal, giant, and supergiant early-type $\mathrm{O}$ stars, which have long been known as X-ray sources (Pallavicini et al. 1981; Sciortino et al. 1990; Güdel \& Nazé 2009). The hypothesis of intrinsic origin for the X-ray emission of BD $+37^{\circ} 1977$ is further supported by the spectral analysis. In fact, considering the likely possibility of non-solar composition, the spectrum can be successfully described by the sum of two thermal plasma components, as in normal early-type stars.

It is interesting to compare the properties of the three sdOs for which X-ray spectral information is available (Table 4). The spectrum of HD 49798 during the eclipse phase can be described by the sum of three thermal plasma components (Mereghetti et al. 2013). While the hottest component is required to account for the significant emission above $\sim 2 \mathrm{keV}$, the temperatures

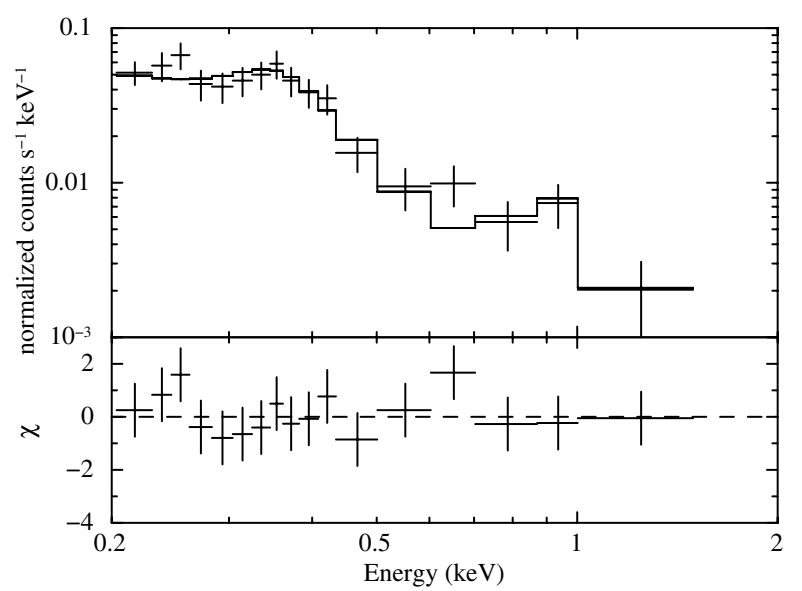

Fig. 3. Top panel: EPIC pn spectrum of $\mathrm{BD}+37^{\circ} 1977$ with the best-fit model composed of the sum of two thermal plasma components, with abundances from Jeffery \& Hamann (2010). Bottom panel: residuals (in units of $\sigma$ ) between data and model.

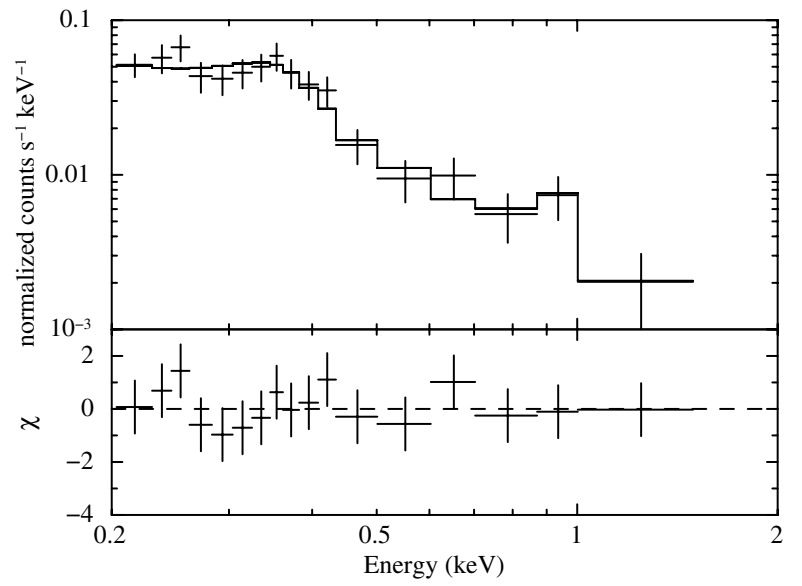

Fig. 4. Top panel: EPIC $p n$ spectrum of $\mathrm{BD}+37^{\circ} 1977$ with the best-fit model composed of the sum of two thermal plasma components, with abundances from Jeffery \& Hamann (2010) and free oxygen abundance. Bottom panel: residuals (in units of $\sigma$ ) between data and model.

of the two coldest components are very similar to those of $\mathrm{BD}+37^{\circ} 1977$. The $0.2-10 \mathrm{keV}$ luminosity of HD 49798 during the eclipse phase is $L_{X} \simeq 3 \times 10^{30} \mathrm{erg} \mathrm{s}^{-1}$, i.e. one order of magnitude lower than that of $\mathrm{BD}+37^{\circ} 1977$. However, because of its lower bolometric luminosity, for HD 49798 the $\mathrm{X}$-ray/bolometric luminosity ratio is also $L_{\mathrm{X}} / L_{\mathrm{bol}} \sim 10^{-7}$. The spectrum of the other X-ray emitting $\mathrm{sdO}$ star, $\mathrm{BD}+37^{\circ} 442$, can also be fit with a similar thermal model with the $\mathrm{He}$ and metal abundances derived for this star (Jeffery \& Hamann 2010) and the temperatures indicated in Table 4. Its flux $f_{\mathrm{X}} \simeq 6.4 \times$ $10^{-14} \mathrm{erg} \mathrm{cm}^{-2} \mathrm{~s}^{-1}$ implies a luminosity $L_{\mathrm{X}} \simeq 2.9 \times 10^{31} \mathrm{erg} \mathrm{s}^{-1}$ (for a distance of $2 \mathrm{kpc}$ ), hence an X-ray/bolometric luminosity ratio $L_{\mathrm{X}} / L_{\mathrm{bol}}=10^{-6.3}$.

These results indicate that the X-ray emission from these three luminous sdO stars is similar to that of normal O-type stars, which have luminosities up to a few $10^{33} \mathrm{erg} \mathrm{s}^{-1}$. In these stars the X-ray emission is due to turbulence and shocks in their strong embedded winds (Lucy \& White 1980; Owocki et al. 1988). Since the mass-loss rate in the radiation-driven winds of early type stars scales with the bolometric luminosity, and the $\mathrm{X}$-ray emission originates in the stellar wind, a correlation between $L_{\mathrm{X}}$ and $L_{\mathrm{bol}}$ is not surprising (see e.g. Owocki et al. 2013). 
Table 4. Summary of the best-fit parameters of the three sdO stars observed with XMM-Newton, when their spectrum is described with multitemperature thermal-plasma components (MEKAL in XSPEC).

\begin{tabular}{cccccc}
\hline \hline Source & $\begin{array}{c}k T_{1} \\
(\mathrm{keV})\end{array}$ & $\begin{array}{c}k T_{2} \\
(\mathrm{keV})\end{array}$ & $\begin{array}{c}k T_{3} \\
(\mathrm{keV})\end{array}$ & $\log \left(L_{\mathrm{X}} / L_{\mathrm{bol}}\right)$ & Reference \\
\hline HD 49798 (in eclipse) & $0.13 \pm 0.02$ & $0.71_{-0.19}^{+0.15}$ & 5 (fix) & -7.1 & Mereghetti et al. (2013) \\
BD $+37^{\circ} 442$ & $0.17_{-0.01}^{+0.02}$ & $0.72_{-0.10}^{+0.22}$ & - & -6.3 & This work \\
BD $+37^{\circ} 1977$ & $0.10_{-0.02}^{+0.04}$ & $0.84_{-0.25}^{+0.57}$ & - & -6.5 & This work \\
\hline
\end{tabular}

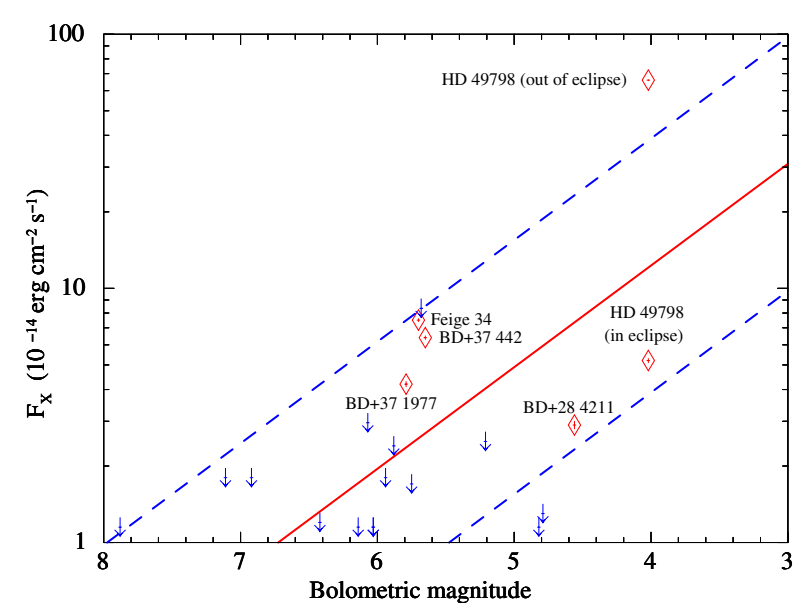

Fig. 5. Level of the X-ray flux (or its upper limit for the undetected sources) of the sdO stars observed at X-rays, as a function of their bolometric magnitude. The upper and lower blue lines (corresponding to $f_{\mathrm{X}} / f_{\text {bol }}=10^{-6.2}$ and $f_{\mathrm{X}} / f_{\text {bol }}=10^{-7.2}$, respectively) include the range of expected values for the main-sequence early-type stars; the red line corresponds to $f_{\mathrm{X}} / f_{\mathrm{bol}}=10^{-6.7}$, which is the best-fit relation found by Nazé (2009) for this type of stars.

In this respect it is interesting to note that the three X-ray emitting sdOs are among the few hot subdwarfs for which evidence of mass loss has been reported (Jeffery \& Hamann 2010). Our results indicate that, even if the winds of sdO stars are rather weak (e.g. $\dot{M}=10^{-8.2} M_{\odot} \mathrm{yr}^{-1}$ for $\mathrm{BD}+37^{\circ} 1977$ according to the estimate of Jeffery \& Hamann 2010), they can produce X-ray emitting shocks as in more luminous O-type stars. In this framework, we note that our findings for sdO stars are supported by the methodology used and the results obtained by Cohen et al. (2014), who investigated the X-ray spectra of O-type stars with very low mass-loss rates.

The latest Chandra detections of sdO stars reinforce the hypothesis that this type of stars is also a class of X-ray sources; in this respect, the detection not only of the luminous stars, but also of the compact stars is very promising. In addition, we also note that in all cases the estimated $L_{\mathrm{X}} / L_{\mathrm{bol}}$ ratio agrees with that found in the heavier early-type stars: therefore also in the compact sdO stars the X-ray emission could be attributed to turbulence and shocks in their winds. In order to provide an overview of all the sdO stars observed at X-rays so far, in Fig. 5 we show, as a function of their bolometric magnitude, the X-ray flux of the detected stars and its upper limit for the undetected stars; for $\mathrm{HD} 49798, \mathrm{BD}+37^{\circ} 442$, and $\mathrm{BD}+37^{\circ} 1977$ the flux value is based on the spectral fit provided by the XMM-Newton data, while for the other sources it is based on the count rate value or limit provided by Chandra HRC-I, assuming an emission spectrum similar to that of $\mathrm{BD}+37^{\circ} 1977$. For comparison, the two dashed lines trace the region corresponding to the typical ratio of X-ray to bolometric flux for the normal O-type stars. The plot shows that almost all the observed stars are within this region, therefore the possible presence of intrinsic X-ray emission also from the stars undetected so far cannot be excluded.

Acknowledgements. This work is based on observations obtained with $X M M-N e w t o n$, an ESA science mission with instruments and contributions directly funded by ESA Member States and NASA. P.E. acknowledges a Fulbright Research Scholar grant administered by the US-Italy Fulbright Commission and is grateful to the Harvard-Smithsonian Center for Astrophysics for hosting him during his Fulbright exchange.

\section{References}

Anders, E., \& Grevesse, N. 1989, Geochim. Cosmochim. Acta, 53, 197 Bauer, F., \& Husfeld, D. 1995, A\&A, 300, 481

Brown, T. M., Sweigart, A. V., Lanz, T., Landsman, W. B., \& Hubeny, I. 2001, ApJ, 562, 368

Cohen, D. H., Wollman, E. E., Leutenegger, M. A., et al. 2014, MNRAS, 439, 908

Darius, J., Giddings, J. R., \& Wilson, R. 1979, in The First Year of IUE, 363

Geier, S. 2015, Astron. Nachr., 336, 437

Güdel, M., \& Nazé, Y. 2009, A\&ARv, 17, 309

Hamann, W. 2010, Ap\&SS, 329, 151

Hamann, W., Gruschinske, J., Kudritzki, R. P., \& Simon, K. P. 1981, A\&A, 104, 249

Heber, U. 2009, ARA\&A, 47, 211

Heber, U., \& Jeffery, C. S. . 1992, The Atmospheres of Early-Type Stars (Berlin: Springer Verlag), Lect. Notes Phys., 401

Heber, U., Hirsch, H., Ströer, A., \& et al. 2006, Balt. Astron., 15, 91

Heber, U., Geier, S., Irrgang, A., et al. 2014, in 6th Meeting on Hot Subdwarf Stars and Related Objects, 481, 307

Hirsch, H. A., Heber, U., \& O'Toole, S. J. 2008, in Hot Subdwarf Stars and Related Objects, eds. U. Heber, C. S. Jeffery, \& R. Napiwotzki, ASP Conf. Ser., 392, 131

Iben, I., Jr. 1990, ApJ, 353, 215

Israel, G. L., Stella, L., Angelini, L., et al. 1997, ApJ, 474, L53

Jeffery, C. S., \& Hamann, W.-R. 2010, MNRAS, 404, 1698

Jordi, C., Figueras, F., Paredes, J. M., Rossello, G., \& Torra, J. 1991, A\&AS, 87, 229

Kudritzki, R. P., \& Simon, K. P. 1978, A\&A, 70, 653

Landolt, A. U. 1973, PASP, 85, 661

Landolt, A. U., \& Uomoto, A. K. 2007, AJ, 133, 768

La Palombara, N., Mereghetti, S., Tiengo, A., \& Esposito, P. 2012, ApJ, 750, L34

La Palombara, N., Esposito, P., Mereghetti, S., \& Tiengo, A. 2014, A\&A, 566, A4

Lucy, L. B., \& White, R. L. 1980, ApJ, 241, 300

Mereghetti, S., Tiengo, A., Esposito, P., \& et al. 2009, Science, 325, 1222

Mereghetti, S., La Palombara, N., Tiengo, A., et al. 2013, A\&A, 553, A46

Napiwotzki, R. 2008, in Hot Subdwarf Stars and Related Objects, eds. U. Heber,

C. S. Jeffery, \& R. Napiwotzki, ASP Conf. Ser., 392, 139

Nazé, Y. 2009, A\&A, 506, 1055

Owocki, S. P., Castor, J. I., \& Rybicki, G. B. 1988, ApJ, 335, 914

Owocki, S. P., Sundqvist, J. O., Cohen, D. H., \& Gayley, K. G. 2013, MNRAS, 429, 3379

Pallavicini, R., Golub, L., Rosner, R., et al. 1981, ApJ, 248, 279

Saio, H., \& Jeffery, C. S. 2000, MNRAS, 313, 671

Saio, H., \& Jeffery, C. S. 2002, MNRAS, 333, 121

Sciortino, S., Vaiana, G. S., Harnden, F. R., Jr., et al. 1990, ApJ, 361, 621

Strüder, L., Briel, U., Dennerl, K., et al. 2001, A\&A, 365, L18

Thej1l, P., MacDonald, J., \& Saffer, R. 1991, A\&A, 248, 448

Turner, M. J. L., Abbey, A., Arnaud, M., et al. 2001, A\&A, 365, L27

Ulla, A., \& Thejll, P. 1998, A\&AS, 132, 1

Wolff, S. C., Pilachowski, C. A., \& Wolstencroft, R. D. 1974, ApJ, 194, L83

Zanin, C., \& Weinberger, R. 1997, Planetary Nebulae, 180, 290 